\title{
Cooperative Flow dynamics in production lines with buffer level dependent production rates
}

\author{
R. Filliger ${ }^{1}$, M.-O. Hongler ${ }^{1}$ \\ EPFL \\ Laboratoire de Production Microtechnique (LPM) \\ Institut de Production et Robotique (IPR) \\ CH-1015 Lausanne \\ Switzerland
}

\begin{abstract}
We study, in the fluid flow framework, the cooperative dynamics of a buffered production line in which the production rate of each work-cell does depend on the content of its adjacent buffers. Such state dependent fluid queueing networks are typical for people based manufacturing systems where human operators adapt their working rates to the observed environment. We unveil a close analogy between the flows delivered by such manufacturing lines and cars in highway traffic where the driving speed is naturally adapted to the actual headway. This close analogy is thoroughly explored. In particular, by investigating the dynamic response of small perturbations around free flow stationary regimes, we can draw a "phase diagram". This diagram exhibits two different flow patterns, namely the free and jamming production regimes. The transitions between these regimes are tuned by the production control parameters (i.e. the buffer capacities, the reaction sensitivity, the control sampling time, ...). We finally extract a dimensionless dynamic parameter directly relevant for design purposes.
\end{abstract}

Key words: buffered production flows, car traffic, state dependent fluid queueing networks, optimal velocity models, linear dynamic response theory, coupled map lattices, free versus jamming transport regimes.

Email addresses: roger.filliger@epfl.ch (R. Filliger), max.hongler@epfl.ch (M.-O. Hongler).

1 In part supported by the "Fonds National Suisse pour la Recherche Scientifique" 


\section{Introduction}

It is already half a century that B. Zimmern [1] wrote a pioneering paper devoted to the characterization of production flows in serial lines. This original contribution has stimulated a strong research activity with the aim to calculate the average throughput and other performance measures for failure prone flowand job-shop manufacturing systems (see [2,3] for recent and comprehensive reviews devoted to this topic). Due to the tremendously growing role played by computers in the seventies and eighties of the $\mathrm{XX}^{\text {th }}$ century, it has long been believed that the ultimate goal in manufacturing would consist in building complete production plants without any human presence. According to this "no-human presence" paradigm, most of the production flow research papers have been oriented towards the study of shop floors equipped with automated machines with rigid behavior (i.e. either the machine is "on" and it delivers with maximal speed or it is "off" and nothing is produced). Today, this nohuman factory is widely questioned and one indeed realizes that the inherent flexibility of human operators offers irreplaceable advantages at reasonable costs. In a very recent address, [4], Chap.3, J. A. Buzacott emphasized this point and delivers the view that the main challenge for future research in production systems should be oriented towards the study of models which incorporate key features like the people incentives and capabilities.

While cooperative dynamics does already occur in purely automated multistages lines (via the starving and blocking mechanisms), the presence of human operators introduces additional adaptability features. While in the purely automated case, the production rates of the work-cells are generically of the "bang-bang type" (i.e produce at the maximal rate when "on" and no production when "off"), human operators usually behave in a much more flexible manner. It is for instance common for human operators to observe the content of their up- and down-stream buffers and then, based on these observations, to suitably regulate (i.e. increase or decrease) their processing rate in order to avoid blocking and/or starving of the flows. This greater flexibility in their dynamics behaviors obviously influences the overall production flows in either a positive or sometimes also a negative way. An illustration is given by J.A. Buzacott ([4] Chap. 3.7) of an automated guided vehicles (AGV) based assembly line in which the operators freely decide when to launch their AGV. The key point here is the possibility for the operators to themselves freely control their idle time (and hence their production rate).

The aim of the present paper is i) to study how the production flows depend on machines with flexible production rates typical in presence of human operators and ii) to show how a synergetic approach can be used to study the production flow dynamics. This is realised by means of a simple modeling framework. The modeling is based on the close analogy which exists between the production 
flows and the flow of cars in highway traffic (see $[5,6]$ for an up-to-date review of traffic theory). The analogy enable us to study analytically some features of the underlying cooperative dynamics. Cooperation gives rise to different flow regimes (i.e. free-flows versus jamming flows) which are tuned according to the values of external control parameters. The parameters range defining the boundary between these flow regimes follow directly and analytically from our synergetic approach.

While the transitions from the free to the jammed flow regimes are well known in the car traffic domain, such transitions have so far received very little attention in the production literature. This is not surprising as, in manufacturing, one usually focus on calculating (stationary) performance measures based on ergodic measures. To study phenomena like transitions from free to jammed flow regimes, one needs information regarding individual realisations of the dynamics rather than the equilibrium statistical mechanical ensembles. When a particular solution of the dynamics (i.e. a realisation) is known, the natural question is to determine the range of control parameters for which it remains stable. A given flow regime is thereby stable when the transient linear response of the system to a time-localized perturbation is damped out. Applying this method to our simple production line, we are able to draw a flow diagram which exhibits transitions from a free to a jammed regime. The transitions are tuned by external control parameters such as the buffer contents the reactivity of the human operators or the sampling time at which the state of the system is monitored.

Our paper is organised as follows: In section 2, we jointly present a production flow shop model and a single lane model for cars in traffic. A direct correspondence between the models is established and differences as well as similarities of the dynamics are discussed. The stability of the flows is analysed in section 3 in the continuous and the discrete time framework. In the former case we construct a dimensionless stability parameter which is recognised to play a central role also in the calculation of the throughput via stationary probability measures. For discrete time, the dynamics is expressed as a coupled-map lattice for which a phase diagram for the flow is derived. Section 4 is devoted to conclusions.

\section{Models}

\subsection{Buffered Flow Shop}

Let us consider a flow shop producing a single final product. The flow shop is made up by $N$ machines in tandem $\left\{M_{k}\right\}_{k=1 . . N}$ separated by $N-1$ buffers 
$\left\{B_{k}\right\}_{k=1 . . N-1}$ as showed in Fig. 1. We adopt here an hydrodynamic point of view and assume that the buffer population can be modeled by a continuous variable.

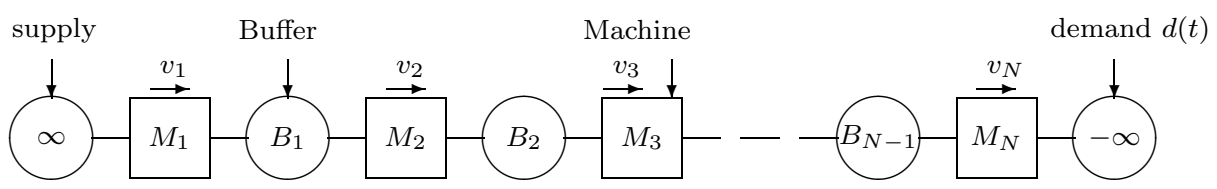

Fig. 1. Sketch of a flow shop composed of $N$ machines and $N-1$ buffers. We suppose perfect supply such that $M_{1}$ is never starved (there is always enough raw materials stored in " $+\infty "$ ") and a high demand rate such that $M_{N}$ is never blocked (i.e. all the final goods are absorbed in " $-\infty$ ").

The buffer $B_{k}$ is located between the machines $M_{k}$ and $M_{k+1}$. The population level of $B_{k}$ at time $t$ is $y_{k}(t) \in \mathbb{R}$. Hence, we shall have $0 \leq y_{k}(t) \leq h_{k}$ where $h_{k}>0$ stands for the capacity of the buffer $B_{k}$. We assume a degree of adaptability, to be defined below, of the production rate $v_{k}$ of machine $M_{k}$. More specifically, we shall assume that $v_{k}$ can vary continuously in the interval $\left[0, V_{\max , k}\right]$, where $V_{\max , k}$ is the maximal production rate of $M_{k}$.

The occasional breakdowns of random duration of the $M_{k}$ 's are modeled by independent Markov processes $I_{1}(t), \ldots, I_{N}(t)$, where $I_{k}(t)$ is an alternating renewal process with exponential holding times in the two states $\{0,1\}$. We say that the machine $M_{k}$ is up whenever $I_{k}(t)=1$ and $M_{k}$ is down when $I_{k}(t)=0$. The $I_{k}$ 's are characterised by the mean up and the mean down times $\lambda_{k}^{-1}$ respectively $\mu_{k}^{-1}$.

For simplicity, the rate of demand $d(t)$ facing the system is from now on assumed to be large enough to systematically absorb the production. Accordingly and for notational ease, we set $v_{N+1}(t)=d(t)$ and $I_{N+1}(t) \equiv 1$. In addition the following assumptions are made:

A1) $y_{k}(t)=0 \Rightarrow v_{k+1}(t) \leq v_{k}(t), k=1, \ldots, N-1$, which means that when the downstream buffer of $M_{k}$ is empty, $M_{k+1}$ is starved. In this case the production rate of $M_{k+1}$ is slaved by the production rate of $M_{k}$.

A2) $y_{k}(t)=h_{k} \Rightarrow v_{k}(t) \leq v_{k+1}(t), k=1, \ldots, N-1$, which means that when the upstream buffer of $M_{k+1}$ is plain, $M_{k}$ is blocked. In this case the production rate of $M_{k}$ is slaved by the production rate of $M_{k+1}$.

A3) Transport time of items from $M_{k}$ to $B_{k}$ and from $B_{k}$ to $M_{k+1}, k=1, \ldots, N-$ 1 , are assumed to be short and will be neglected.

A4) Machine $M_{1}$ is never starved (enough raw material) and $M_{N}$ is not influenced by the market (enough demand).

The assumption A4 simplifies the "boundary" conditions and the assumptions A1-A3 can be summarised as follows: "while operating at time $t$ and as long 
as $M_{k}$ is neither starved nor blocked, $M_{k}$ can produce continuously and independently of the other machines with the production rate $v_{k}(t)$ and its output is instantaneously stored in $B_{k}$." The evolution of the inventories can then be written as follows:

$$
\frac{\mathrm{d} y_{k}(t)}{\mathrm{d} t}=v_{k}(t) I_{k}(t)-v_{k+1}(t) I_{k+1}(t), \quad y_{k}(0)=y_{k}, \quad k=1, \ldots, N,
$$

where the $y_{k}$ 's are fixed initial conditions with $y_{k} \in\left[0, h_{k}\right]$. The inventories are naturally subject to the state constraints

$$
\left(y_{1}(t), \ldots, y_{N-1}(t), y_{N}(t)\right) \in\left[0, h_{1}\right] \times \ldots \times\left[0, h_{N-1}\right] \times \mathbb{R}
$$

which must be satisfied at every instant. To complete the dynamics of the flow shop model one has to specify the production rates $v_{k}$ of the machines subject to the constraints:

$$
\left(v_{1}(t), \ldots, v_{N}(t)\right) \in\left[0, V_{\max , 1}\right] \times \ldots \times\left[0, V_{\max , N}\right] .
$$

This is customarily done (at least in automated systems literature) by expressing the production rates as being the solutions of a convex production planning model which minimizes a specific cost functional (see e.g., [7]). Here we are not dealing with such an optimisation problem but we are interested in the dynamical response of a flow shop with state dependent production rates to a perturbation. Therefore we fix the dynamics (i.e. the production rates) by choosing a class of production rate functions which take into account (2) and (3). For this purpose it is now time to introduce below the so called "optimalvelocity" car traffic model first studied by M. Bando et al. [8] in analogy to which we will derive the production rates.

\subsection{Optimal-Velocity Car Traffic Model}

We consider $N$ cars $\left\{M_{k}\right\}_{k=1 . . N}$ traveling on a single lane as showed in Fig. 2 . For $k=1, \ldots, N-1$, denote by $x_{k}(t)>0$ the headway between the cars

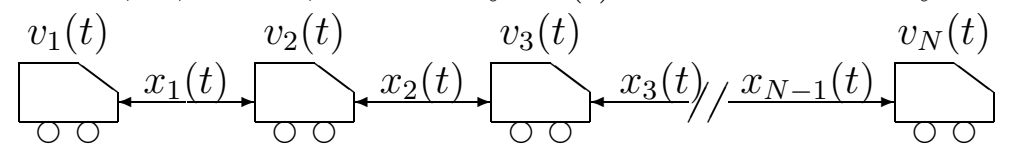

Fig. 2. $N$ cars on a single-lane.

$M_{k}, M_{k+1}$ and for $k=1, \ldots, N$ denote by $v_{k}(t) \in\left[0, V_{\max , k}\right]$ the speed of $M_{k}$, where $V_{\max , k}$ is the maximal velocity of $M_{k}$.

The Optimal Velocity (OV) traffic model [8] states the existence of an optimal velocity function $\mathcal{V}_{k}$ which depends on the headways $x_{k}, x_{k-1}$ and the presence 
of a response delay time $\tau_{k}$, required for a driver of $M_{k}$ to adjust its speed, such that:

$$
\mathcal{V}_{k}(t) \stackrel{\text { not. }}{=} \mathcal{V}_{k}\left(x_{k-1}(t), x_{k}(t)\right)=v_{k}\left(t+\tau_{k}\right)
$$

Expanding Eq.(4) up to first order, adding the corresponding headway variations and specifying the optimal velocity yields the following class of OVmodels:

$$
\left\{\begin{array}{l}
\frac{\mathrm{d} x_{k}(t)}{\mathrm{d} t}=v_{k+1}(t)-v_{k}(t), \quad k=1, \ldots, N \\
\frac{\mathrm{d} v_{k}(t)}{\mathrm{d} t}=\alpha_{k}\left(\mathcal{V}_{k}(t)-v_{k}(t)\right), \quad k=1, \ldots, N
\end{array}\right.
$$

where $\alpha_{k}=\tau_{k}^{-1}$ and where the optimal velocity of $M_{k}$ at time $t$ is of the form:

$$
\mathcal{V}_{k}(t)=V_{\max , k} \cdot F_{k}\left(x_{k-1}(t), x_{k}(t)\right)
$$

In writing Eqs.(5) and (6), the following set of control parameters and functions are introduced:

a) The set of parameters $\alpha_{k}>0$ which have the physical dimension of frequencies. They represent the sensitivity of the control mechanisms acting on the speed of the cars and are given by the inverse of the delay times $\tau_{k}$ introduced in (4) (i.e. $\alpha_{k}=\tau_{k}^{-1}$ ).

b) The set of functions $F_{k}$, associating to given up- and downstream headways $x_{k-1}$ and $x_{k}$ a dimensionless factor $F_{k}\left(x_{k-1}, x_{k}\right) \in[0,1]$. When multiplied by the maximum speed $V_{\max , k}$ of $M_{k}, F_{k}$ yields the optimal velocity of $M_{k}$. The functions $F_{k}$ model directly the speed adaptability of $M_{k}$ as a function of the headway to its adjacent neighbors. The type of controls that the $F_{k}$ 's introduce, are similar to those considered in [9] to describe the psychological effects of car drivers.

Note that the functions $F_{k}$ are, and hence so are the OV-functions, of phenomenological nature and have to be chosen by the model builder. Based on common intuition the functions $F_{k}(x, y)$ should be non-increasing in the upstream variable $x$ (no tendency to accelerate if the upstream headway increases) and non-decreasing in the downstream variable $y$ (no tendency to brake down if the downstream headway increases). For calculation purposes we shall assume, from now on, that the $F_{k}$ 's are at least twice continuously differentiable in $x$ and $y$. Note that various explicit choices for OV-functions are used in traffic theory. They are guided by criteria like simplicity and existence of explicit solutions, low number of control parameters, existence of inflection points...[8-10]. 


\subsection{Correspondence between Flow Shops and Optimal-Velocity models}

A glance at Figs. (1) and (2) and at Eqs. (1) and (5), suggests the following correspondence:

$$
\begin{aligned}
& \text { machines in a flow shop } \leftrightarrow \quad \text { cars in a single lane, } \\
& \text { free buffer space } h_{k}-y_{k} \leftrightarrow \quad \text { headway } x_{k} \text {, } \\
& \text { production rate } \quad \leftrightarrow \quad \text { car velocity, }
\end{aligned}
$$

from where we deduce, keeping failure prone machines, that an optimal production rate flow shop model can be written as:

$$
\left\{\begin{array}{l}
\frac{\mathrm{d} y_{k}(t)}{\mathrm{d} t}=v_{k}(t) I_{k}(t)-v_{k+1}(t) I_{k+1}(t), \quad k=1, \ldots, N, \\
\frac{\mathrm{d} v_{k}(t)}{\mathrm{d} t}=\alpha_{k}\left(V_{\max , k} \cdot \phi_{k}\left(y_{k-1}(t), y_{k}(t)\right)-v_{k}(t) I_{k}(t)\right) .
\end{array}\right.
$$

where the $\phi_{k}$ 's are directly related to the $F_{k}$ 's by

$$
\phi_{k}(x, y):=F_{k}\left(h_{k-1}-x, h_{k}-y\right)
$$

thereby realising the correspondence "free buffer space = headway".

In this setting, the functions $\phi_{k}(x, y)$ represent for fixed $x$ the influence of the free downstream buffer space on the production rate of $M_{k}$ and similarly for fixed $y$, one gets the influence of the free upstream buffer space on the production rate of $M_{k}$. In particular, the starving resp. the blocking mechanisms of $M_{k}$ are taken into account by imposing $\phi_{k}\left(h_{k-1}, y\right)=F_{k}\left(0, h_{k}-y\right)=0$ resp. $\phi_{k}(x, 0)=F_{k}\left(h_{k-1}-x, h_{k}\right)=0$. Indeed, when $M_{k}$ is starved (resp. blocked) the free upstream buffer space equals $h_{k-1}$ (the free downstream buffer space equals 0$)$ and we have $\phi_{k}\left(h_{k-1}, y\right)=0$ independent of the downstream buffer variable $y$ (resp. $\phi_{k}\left(x_{k-1}, 0\right)=0$ independent of the upstream buffer variable $x)$. Eq.(7) therefore implies $v_{k}(t)=\exp \left(-\alpha_{k} t\right)$. Hence, in both cases (starved resp. blocked) the production rate of $M_{k}$ decreases exponentially fast. For $\alpha \gg 1$, this behaviour closely approaches the assumption A1: $v_{k}(t) \leq v_{k-1}(t)$ (resp. A2: $v_{k}(t) \leq v_{k+1}(t)$ ). In the limit when $\alpha_{k} \rightarrow \infty$ one effectively has a "bang-bang" type of reaction and the velocity is immediately adjusted to its optimum. In this limiting case the assumptions made on $F_{k}$ takes automatically into account the buffer level constraints expressed in Eq.(2) and Eq.(3). Note that $\mathbf{A} 4$ can be satisfied by imposing $\phi_{1}\left(x, x_{1}\right)=\phi_{1}\left(x_{1}\right)$ and $\phi_{N}\left(x_{N-1}, x_{N}\right)=\phi_{N}\left(x_{N-1}\right)$ which reflects the facts that $M_{1}$ is never starved (independent of the supply) and that $M_{N}$ is never blocked (independent of the market). 
Using the mentioned correspondence, the resulting optimal production rate flow shop model can be summarised as follows: to each machine $M_{k}$ and at every time instant $t$, one can assign an optimal production rate $\mathcal{V}_{k}(t)$ which depends on the up- and downstream buffers of $M_{k}$. This optimal production rate will be reached within a time interval of length $\tau_{k}$ thanks to the adaptable production rate of the operators.

\section{Remarks.}

a) Depending only on $x_{k-1}$ and $x_{k}$, the production rate of $M_{k}$ is constantly adjusted by an operator who monitors the population levels in the up- and downstream buffers. It is better to produce with the optimal production rate $\mathcal{V}_{k}$ but deviations may exist between the optimal- and the real production rate. The operator tries to reduce the deviation $\Delta=\mathcal{V}_{k}-v_{k}$ by initiating an acceleration $\alpha_{k} \Delta$ of its production rate. This response mechanism replaces the state constraints given by Eqs.(2) and (3).

b) The analogy between manufacturing- and traffic systems has its limits. In particular, to breakdowns of machines will correspond sudden stops of vehicles. Such "unitary crashes" are rather rare events on highways. In this sense the OV-traffic model (5) is a special case of the optimal production rate model (7). Indeed, setting $I_{k}=1$ and removing the starving mechanism A1)- absent in real traffic - we obtain exactly the OV-model (5) of Bando et al.. At first sight, one could argue that focusing on $I_{k}=1$ for production lines is not relevant. Indeed for fully reliable machines, one barely sees the need for introducing buffer places. This is however not so here. Indeed:

i) due to human presence, the production rates are not strictly constant but may fluctuate around their averages. The very presence of these fluctuations restore the importance of buffers as they increase the "compressibility" of the production flow. The transient response to a local fluctuation of the production rate will be given by our model.

ii) In presence of adaptable production rates, the ability of the system to respond to a non ideal production state which results after a failure is an essential dynamic performance factor. Hence the study of the transient response of the system to a state configuration out of the ideal one is essential.

\section{Linear stability analysis}

Obviously, a steady state for cars in a line is given when all of them run orderly with the same constant optimal velocity $\mathcal{V}_{k}=v^{e}$ and with constant headway 
$x_{k}^{e}$, such that:

$$
V_{\max , k} F_{k}\left(x_{k-1}^{e}, x_{k}^{e}\right)=v^{e} \quad k=1, \ldots, N-1 .
$$

Here, we focus on the dynamic response of the flow shop to a single, timelocalised perturbation of the free flow regime. We consider a time interval on which $I_{k}=1, k=1, \ldots, N$ i.e., on which all machines are operational. During such time intervals a steady state $\left(x_{1}^{e}, \ldots, x_{N-1}^{e}, v^{e}\right)$ satisfying eq.(9), corresponds to a flow shop configuration where all stages have constant production rates $v^{e}$ and where the buffers maintain the constant levels $\left(h_{1}-x_{1}^{e}, \ldots, h_{N-1}-\right.$ $x_{N-1}^{e}$ ) (recall that $x_{i}=h_{i}-y_{i}$ where $y_{i}$ is the buffer level). We shall call this state a free flow production regime when localised perturbations are damped out. To formalise this definition we set

$$
r_{k}(t):=\sum_{j=0}^{k-1} x_{j}(t) \quad k=1, \ldots, N, \quad x_{0} \equiv 0 .
$$

for the "absolute distance" of "car" $M_{k}$ from the first car $M_{1}$ and undertake the stability analysis of (5) which reads now:

$$
\left\{\begin{array}{l}
\frac{\mathrm{d} r_{k}(t)}{\mathrm{d} t}=v_{k}(t), \mathrm{k}=1, \ldots, \mathrm{N} \\
\frac{\mathrm{d} v_{k}(t)}{\mathrm{d} t}=\alpha_{k}\left(V_{\max , k} F_{k}\left(r_{k}(t)-r_{k-1}(t), r_{k+1}(t)-r_{k}(t)\right)-v_{k}(t)\right) .
\end{array}\right.
$$

\subsection{Time-continuous Analysis}

Assume that $M_{N}$ produces with a constant rate $v^{e}:=\min \left\{V_{\max , k} \mid k=\right.$ $1, \ldots, N\}$. The dynamics (11) has the following steady state:

$$
\left\{\begin{array}{l}
r_{k}^{e}(t)=\sum_{j=1}^{k-1} x_{j}^{e}+v^{e} t \\
v_{k}^{e}(t)=\mathcal{V}_{k}(t)=v^{e}
\end{array}\right.
$$

where the $x_{k}^{e}$ 's satisfy eq.(9). To infer on the stability of the system we introduce a small perturbation $\delta r_{k}(t) \ll r_{k}^{e}(t)$ :

$$
\delta r_{k}(t):=r_{k}(t)-r_{k}^{e}(t)
$$

Linearising (11) around the steady state, we obtain the dynamical response equation:

$$
\frac{\mathrm{d}^{2} \delta r_{k}(t)}{\mathrm{d} t^{2}}=\alpha_{k}\left[V_{\max , k}\left(\delta r_{k+1} \partial_{y} F_{k}+\delta r_{k}\left(\partial_{x} F_{k}-\partial_{y} F_{k}\right)-\delta r_{k-1} \partial_{x} F_{k}\right)-\frac{\mathrm{d} \delta r_{k}}{\mathrm{~d} t}\right]
$$


where

$$
\partial_{x} F_{k}:=\left.\frac{\partial F_{k}(x, y)}{\partial x}\right|_{x=x_{k-1}^{e}} \text { and } \partial_{y} F_{k}:=\left.\frac{\partial F_{k}(x, y)}{\partial y}\right|_{y=x_{k}^{e}} .
$$

In expanding in a discrete Fourier series:

$$
\delta r_{k}(t):=\frac{1}{N} \sum_{j=0}^{N-1} c_{j} e^{2 \pi i \cdot j \frac{k}{N}} e^{(\lambda(j)-i \omega(j)) t}, \quad k \in\{1, \ldots, N\},
$$

where $i^{2}=-1$, the set of dynamical equations (13) yields for every $j \in$ $\{0, \ldots, N-1\}$ the characteristic relation:

$$
P_{k}(\lambda-i \omega) \delta r_{k}+\alpha_{k} V_{\max , k}\left(\partial_{x} F_{k} \delta r_{k-1}-\partial_{y} F_{k} \delta r_{k+1}\right)=0
$$

where we have omitted the $j$ dependance of $\lambda$ and $\omega$ and where

$$
P_{k}(\lambda-i \omega):=(\lambda-i \omega)^{2}+\alpha_{k}(\lambda-i \omega)+\alpha_{k} V_{\max , k}\left(\partial_{y} F_{k}-\partial_{x} F_{k}\right)
$$

Any solution $\lambda(j)-i \omega(j)$ of $(16)$ with $\lambda(j)>0$ gives rise to a growing evolution of the perturbation and hence the initial state is not stable. The stability is given when $\lambda(j)<0$, for $j=1, \ldots, N-1,(j=0$ corresponds to the neutral mode). This yields the condition (see also [9] and Chapt. III/A.2 in [6]):

$$
\frac{V_{\max , k} \cdot\left(\partial_{x} F_{k}+\partial_{y} F_{k}\right)^{2}}{\alpha_{k} \cdot\left(\partial_{y} F_{k}-\partial_{x} F_{k}\right)}<\frac{1}{1+\cos \left(\frac{2 \pi j}{N}\right)} .
$$

Thus, the most unstable mode is realised for $\frac{j}{N} \rightarrow 0$. Accordingly stability will be guaranteed provided:

$$
\frac{\alpha_{k}\left(\partial_{y} F_{k}-\partial_{x} F_{k}\right)}{V_{\max , k} \cdot\left(\partial_{x} F_{k}+\partial_{y} F_{k}\right)^{2}}>2 \forall k \in\{1, \ldots, N\}
$$

We can now directly transfer this dimensionless stability condition to the flow shop model (note that $\partial_{y} \phi_{k}(x, y)=-\partial_{y} F_{k}(x, y)$ and $\partial_{x} \phi_{k}(x, y)=-\partial_{x} F_{k}(x, y)$ ):

$$
\frac{\alpha_{k}\left(\partial_{x} \phi_{k}-\partial_{y} \phi_{k}\right)}{V_{\max , k} \cdot\left(\partial_{x} \phi_{k}+\partial_{y} \phi_{k}\right)^{2}}>2 \forall k \in\{1, \ldots, N\}
$$

\section{Remarks}

1) When (19) is satisfied for all $k=1, \ldots, N$, the variation of velocity of the upstream machine is damped out by the presence of the buffers and the flow shop is running in a soft regime. When (19) fails for some $k$, the regime of "jamming" or "chattering" may occur. This jamming flows are characterised by large fluctuations in the buffer population and hence the machines are likely to be found in a starved or blocked state. 
2) In close analogy with fluid mechanics where the Reynolds' number (determining whether a flow is laminar or turbulent) is used for design purpose, the dimensionless stability criterion given in eq.(19) suggests that the number:

$$
\mathcal{Z}:=\frac{\alpha\left(\partial_{x} \mathcal{V}-\partial_{y} \mathcal{V}\right)}{\left(\partial_{x} \mathcal{V}+\partial_{y} \mathcal{V}\right)^{2}}
$$

is of direct interest for the modeling and design of serial production lines with environment dependent optimal production rates.

3) For a "symmetric" control (i.e., $\partial_{x} \phi_{k}+\partial_{y} \phi_{k}=0$ ) the fundamental inequality (19) is always satisfied and hence the steady state (12) of system (11) is unconditionally stable.

4) The stability of the steady state depends (implicitly) on the buffer capacities via $\partial_{x} \phi_{k}$ and $\partial_{y} \phi_{k}$. To further illustrate this point, let us investigate an explicit case:

Example. Let us consider the case of a production line where in order to minimize work in process, the famous just in time philosophy is applied. Just in time advocates pull production control which says that inventories are not processed until there is adequate space in the next downstream buffer. Hence no upstream control is imposed (i.e. $\partial_{x} \phi_{k}=0$ ). We further suppose that the $\phi_{k}$ 's are independent of $k$ and set:

$$
\phi_{k}\left(x_{k-1}(t), x_{k}(t)\right)=\phi\left(x_{k}(t)\right)= \begin{cases}1 & \text { if } x_{k}(t)=0 \\ 1-\frac{x_{k}(t)}{h_{k}} & \text { if } 0<x_{k}(t)<h_{k} \\ 0 & \text { if } x_{k}(t) \geq h_{k} .\end{cases}
$$

Using Eq.(21), the relation (19) reduces to:

$$
\mathcal{Z}_{k}:=\frac{\alpha_{k} h_{k}}{V_{\max , k}}>2 \quad \forall k \in\{1, \ldots, N\}
$$

or equivalently

$$
\frac{1}{2} \frac{h_{k}}{V_{\max , k}}>\frac{1}{\alpha_{k}}=\tau_{k} \forall k \in\{1, \ldots, N\} .
$$

Hence for stability, the reaction time has to be shorter than twice the time required to empty a filled buffer. Estimating the reaction time of the operators the simple and intuitive condition eq.(23) can be used to determine the buffer capacities.

At this stage, it is worthwhile to bridge, at least partially, the conceptual gap between the stability relation (22) and the behaviour of the stationary throughput $\langle t\rangle$ delivered by a production line. Remember that one method 
to estimate the average throughput of a flow shop relies on the aggregation methods based on the throughput delivered by two stage production lines (i.e. production dipoles). For a production dipole with identical and operation dependent failure prone machines the stationary measure analysis yields (see e.g., [11] p.71 and [12]):

$$
\langle t\rangle=V_{\max } \frac{1}{1+I_{\mathrm{eff}}}, \quad \text { with } I:=\frac{\mu}{\lambda} \leq I_{\mathrm{eff}}:=I\left[1+\frac{2}{2+\mathcal{F}(1+I)}\right] \leq 2 I,
$$

where $\lambda$ (resp. $\mu$ ) is the mean time to failure (resp. reparation), $I$ ( $\left.I_{\text {eff }}\right)$ the (effective) unavailability factor of the dipole, $V_{\max }$ the common production rate and where $\mathcal{F}=\frac{\mu h}{V_{\max }}$ is a dimensionless performance parameter introduced in [11]. Note that one can write:

$$
1 / \mathcal{F}=\frac{1+I}{2}\left(I_{\text {eff }}-I\right) /\left(2 I-I_{\text {eff }}\right),
$$

which is the expected effective unavailability decrease due to an increase of the buffer capacity $h$ (see Figure 3 ). The dimensionless parameter $\mathcal{Z}_{k}$ given in

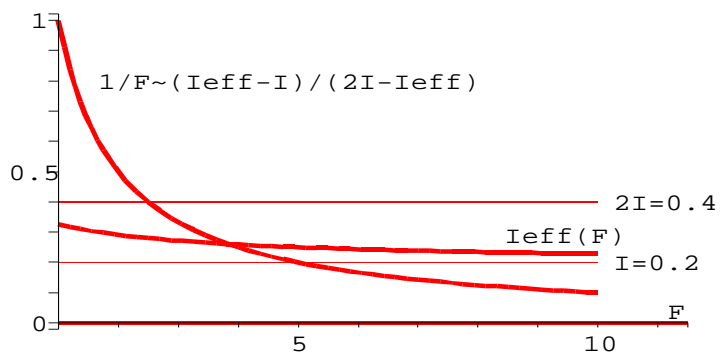

Fig. 3. Sketch of $1 / \mathcal{F}$ and $I_{\text {eff }}(\mathcal{F})$. As $\mathcal{F} \propto h$, an increase of $h$ reduces $I_{\text {eff }}(\mathcal{F})$ and hence enhances the average throughput $\langle t\rangle$. Note that the increase of $\langle t\rangle$ which is very rapid for small $h$ becomes very gradual for larger $h$ and is therefore less rewarding. By identifying the role played by $\mu_{k}$ (reparation rate in the failure prone production lines) and $\alpha_{k}$ (sensitivity in the human based lines), the stability condition given in Eq.(25) shows that the significant gain in production throughput are achieved by avoiding the jamming regimes.

$(22)$ is directly related to $\mathcal{F}$ via:

$$
\frac{1}{\mathcal{F}}=\frac{\alpha_{k}}{\mu_{k}} \frac{1}{\mathcal{Z}_{k}}<\frac{\alpha_{k}}{\mu_{k}} \frac{1}{2} .
$$

Equation (25) relates the stationary (i.e. the expected) effective unavailability decrease with the dimensionless number $\mathcal{Z}_{k}$ which is derived on the basis of a dynamical linear response analysis. Requiring stability for the transient response to a perturbation Eq.(25) implies a lower (upper) bound for the parameter $\mathcal{F},(1 / \mathcal{F})$. Therefore in view of Eq. $(25)$, we see that the increase of the average throughput -which results when increasing $\mathcal{F}$ - can be interpreted as the ability of the production system to quickly absorb local perturbations 
(the characteristic time is given by the parameter $\alpha$ ). Hence Eq.(25) establishes an enlightening connection between the properties of a single realisation of the dynamics (transient performance measures) with those resulting from stationary statistical ensemble averages (stationary performance measure).

Taking $\mathcal{F}=2$ is common practice at the shopfloor level and with eq.(25) this leads to:

$$
\frac{\alpha_{k}}{\mu}>1 \forall k \in\{1, \ldots, N\} .
$$

We therefore see that stability is ensured when the reaction time $1 / \alpha$ is smaller than the mean time needed for machine reparation $1 / \mu$.

\subsection{Discrete time analysis}

In practice it is likely that the state of the system will not be continuously monitored in time but rather on a discrete basis. Due to this time sampling, the production rate will itself be adapted only at discrete times. Let us now study this situation by considering the discrete analog of the above analysis. This is done by choosing a sampling time $T>0$ and by updating the system (5) at time-instants $n T, \quad n \in \mathbb{N}$. The resulting model has the form of a coupled map lattice and reads for $k=1, \ldots, N$ :

$$
\left\{\begin{array}{l}
x_{k}(n+1)=x_{k}(n)+\left[v_{k+1}(n)-v_{k}(n)\right] T \\
v_{k}(n+1)=v_{k}(n)+\alpha_{k}\left[V_{\max , k} \cdot F_{k}\left(x_{k-1}(n), x_{k}(n)\right)-v_{k}(n)\right] T
\end{array}\right.
$$

where we write $f(n):=f(n T)$ with $n \in \mathbb{N}$ for an arbitrary function of time $f(t)$. As in the continues case, we derive from Eqs.(27) a stationary regime:

$$
x_{k}(n)=x_{k}^{e} \text { and } v_{k}(n)=v^{e} k=1, \ldots, N,
$$

provided that $x_{k}^{e}$ and $v^{e}$ satisfies the relations eq.(9). Adding a perturbation term $\delta x_{k}$ and linearising around the steady state gives:

$$
\begin{gathered}
\delta x_{k}(n+1)=\delta x_{k}(n)+\left[\delta v_{k+1}(n)-\delta v_{k}(n)\right] T \\
\delta v_{k}(n+1)=\left(1-\alpha_{k} T\right) \delta v_{k}(n)+\alpha_{k} V_{\max , k}\left[\partial_{x} F_{k}\left(x_{k-1}^{e}\right) \delta x_{k-1}+\partial_{y} F_{k}\left(x_{k}^{e}\right) \delta x_{k}\right] T,
\end{gathered}
$$

where $\partial_{x} F_{k}\left(x_{k-1}^{e}\right)=: \partial_{x} F_{k}$ and $\partial_{y} F_{k}\left(x_{k}^{e}\right)=: \partial_{y} F_{k}$ are defined as in Eq.(14).

To simplify the analysis of the above equations, we suppose $\partial_{x} F_{k} \equiv 0$; i.e. there is no dependance of the production rate on the upstream buffer. Note 
that the inequalities

$$
\frac{\alpha_{k} \cdot\left(\partial_{y} F_{k}-\partial_{x} F_{k}\right)}{V_{\max , k} \cdot\left(\partial_{x} F_{k}+\partial_{y} F_{k}\right)^{2}} \geq \frac{\alpha_{k} \cdot\left(\partial_{y} F_{k}-0\right)}{V_{\max , k} \cdot\left(\partial_{y} F_{k}+0\right)^{2}} \geq 2
$$

imply that systems which are stable without upstream controls $\left(\partial_{x} F_{k} \equiv 0\right)$ remain stable when a monotone upstream control $\left(\partial_{x} F_{k}\left(x, x_{k}\right) \leq 0\right)$ is added. Therefore, the subsequent calculations, performed with the auxiliary assumption $\partial_{x} F_{k} \equiv 0$, will still give relevant stability criterions.

Under this assumption, Eqs.(28) and (29) take the canonical form of a discrete feedback system [13]:

$$
\begin{gathered}
\left(\begin{array}{c}
\delta v_{k}(n+1) \\
\delta x_{k}(n+1)
\end{array}\right)=\left(\begin{array}{cc}
1-\alpha_{k} T & \alpha_{k} V_{\max , k} T \partial_{y} F_{k} \\
-T & 1
\end{array}\right)\left(\begin{array}{l}
\delta v_{k}(n) \\
\delta x_{k}(n)
\end{array}\right)+\left(\begin{array}{c}
0 \\
T
\end{array}\right) \delta v_{k+1}(n), \\
\delta v_{k}(n)=\left(\begin{array}{ll}
1 & 0
\end{array}\right)\left(\begin{array}{l}
\delta v_{k}(n) \\
\delta x_{k}(n)
\end{array}\right)
\end{gathered}
$$

The behavior of the above discrete time linear system is analysed via the transfer function $G_{k}(z)$ from $\delta v_{k+1}(n)$ to $\delta v_{k}(n)$ :

$$
G_{k}(z)=\left(\begin{array}{ll}
1 & 0
\end{array}\right) H_{k}(z)^{-1}\left(\begin{array}{ll}
0 & T
\end{array}\right)
$$

with the definition

$$
H_{k}(z):=\left(\begin{array}{cc}
\alpha_{k} T+z-1 & -\alpha_{k} V_{\max , k} T \partial_{y} F_{k} \\
T & z-1
\end{array}\right) .
$$

Stability condition. The stability of the steady state is achieved when the roots of the characteristic equation

$$
\operatorname{det}\left(H_{k}(z)\right)=z^{2}+z\left(\alpha_{k} T-2\right)+\left[1-\alpha_{k} T+\alpha_{k} V_{\max , k} T^{2} \partial_{y} F_{k}\right]=0
$$

lie inside the unit circle. The use of the Schur-Cohn criterion for Eq.(35), (see for instance pp. 56 in [14]) directly implies:

Stability $\Leftrightarrow\left\{\begin{array}{l}0<V_{\max , k}\left|\partial_{y} F_{k}\right| T<1 \\ 0<\alpha_{k} T<\frac{4}{2-V_{\max , k} T\left|\partial_{y} F_{k}\right|}\end{array} \Leftrightarrow\left\{\begin{array}{l}0<V_{\max , k}\left|\partial_{y} F_{k}\right|<1 / T \\ 0<\alpha_{k}<4 / T .\end{array}\right.\right.$

No-jamming condition. The global criterion attenuating velocity disturbances along the production line uses the so-called $H_{\infty}$-norm of the transfer 
matrixes $G_{k}$ and is given by:

$$
\max _{|z|=1}\left|G_{k}(z)\right| \leq 1 \quad \forall k \in\{1, \ldots, N-1\} .
$$

Using Eqs.(33) and (36) together with lengthy algebra, the no-jamming condition (37) can be rewritten as (see also, [13]):

$$
\max \left(0, \frac{1}{T} \frac{8+\alpha_{k} T\left(\alpha_{k} T-8\right)}{\alpha_{k} T\left(\alpha_{k} T-6\right)}\right) \leq V_{\max , k}\left|\partial_{y} F_{k}\right| \leq \frac{\alpha_{k}}{2+\alpha_{k} T} .
$$

Using $\left|\partial_{y} \phi_{k}(x, y)\right|=\left|\partial_{y} F_{k}(x, y)\right|$, we can reformulate the no-jamming condition for the flow shop model (7):

$$
\max \left(0, \frac{1}{T} \frac{8+\alpha_{k} T\left(\alpha_{k} T-8\right)}{\alpha_{k} T\left(\alpha_{k} T-6\right)}\right) \leq V_{\max , k}\left|\partial_{y} \phi_{k}\right| \leq \frac{\alpha_{k}}{2+\alpha_{k} T}
$$

or equivalently in terms of the dimensionless stability parameters $X_{k}=\alpha_{k} T$ and $\mathcal{Z}_{k}:=-\frac{\alpha_{k}}{V_{\max , k} \partial_{y} \phi_{k}}=\frac{\alpha_{k}}{V_{\max , k}\left|\partial_{y} \phi_{k}\right|}$ :

$$
\max \left(0, \frac{1}{X_{k}} \frac{8+X_{k}\left(X_{k}-8\right)}{X_{k}\left(X_{k}-6\right)}\right) \leq \frac{1}{\mathcal{Z}_{k}} \leq \frac{1}{2+X_{k}} .
$$

The region for a soft-running regime (i.e. a free-flow traffic) defined by condition (39) is sketched in the flow diagram Fig. 4 for different sampling times $T=4,2$ and 1.5. The $x$ - and $y$ - axes are resp. spanned by the control parameters $\alpha$ and $V_{\max , k}\left|\partial_{y} \phi_{k}\right|$. The Figure (4) shows the influence of an increasing sampling time $T$ on the soft-running regime given by (39). It clearly exhibits that when the state of the system is less frequently monitored the control parameters must be chosen more carefully to guarantee a soft-running production flow (homogeneous flow). Note that for $T \rightarrow 0$ (i.e continuous monitoring of the system states) condition (39) consistently coincides with the time continuous stability relation (19) (remember that by assumption, $\partial_{x} \phi_{k}=0$ ). A dimensionless interpretation of the soft-running regime is sketched in the flow diagram Fig.(5) which is based on the condition (40). Here the $x$ - and $y$-axes are resp. spanned by the dimensionless parameters $\alpha T$ and $1 / \mathcal{Z}$. The figure (5) illustrates that above a critical value for $\alpha T$ no homogeneous production flow can be expected.

\section{Conclusions}

Among the numerous performance characteristics that modern production systems have to fulfill, the ability to quickly react to sudden and often unexpected changes of the environment is nowadays considered to be the most 


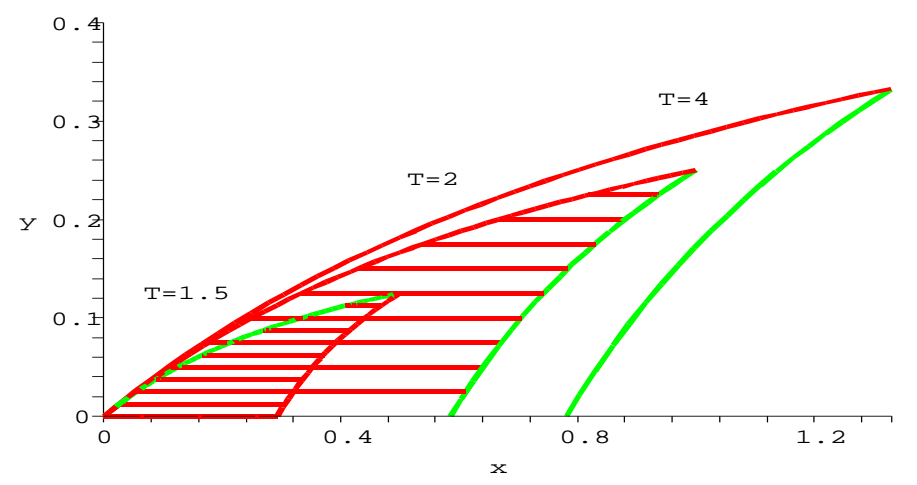

Fig. 4. Sketch of the free flow production regions enclosed by the $x$-axes and: $\frac{2 / T+x(T x-8)}{T x(T x-6)} \leq y \leq \frac{x}{2+T x}$, for $T=4,2$ and $T=1.5$. The $x$ - and $y$-axis represent respectively the sensitivity $x=\alpha$ and the parameter $y=V_{\max , k}\left|\partial_{y} \phi_{k}\right|$. The free-flow regions decrease with increasing sampling times $T$ and vanish for $T \rightarrow \infty$.

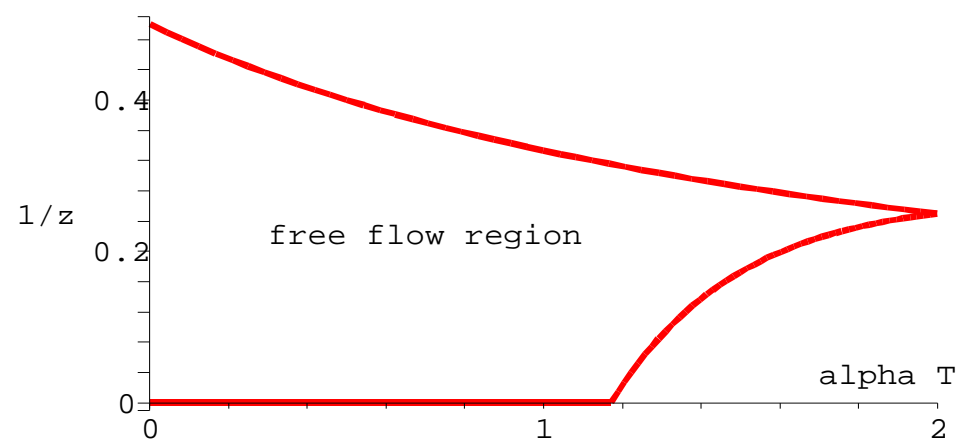

Fig. 5. Sketch of the free flow production region using the dimensionless quantities $\alpha T$ ( $x$-axis) and $1 / \mathcal{Z}$ (y-axis) delimited by the $x$-axis and the eq.(40). Note that no free-flow can be expected if $\alpha T>2$. For $\alpha T=0$ the free-flow condition equals the time continuous stability condition $\mathcal{Z}>2$.

crucial. It has been realized that his time dependant flexibility requirement can often be achieved only at the expense of introducing human operators in the production process. The presence of human operators together with changing environments into a production line strongly complexifies the modeling of the production flows. In particular, stationary performance measures alone are not enough to suitably characterize the production flows and the knowledge of the transient response of the system to fluctuations becomes mandatory. The central role played by transient phenomena is obviously not restricted to production. Indeed, since about a half of century, the ubiquitous presence of transient regimes in vehicular traffic has stimulated an important research activity which produces a wealth of methods and results developed for their understanding. These methods were hardly so far being used in the production flow context. In this paper, we have adopted a synergetic view to explore some of the analogies between simple car traffic models and production lines in which the production rates depend on the contents of adjacent 
buffers. Thanks to a suitable dictionary, we are able to identify production flow regimes which are realised for definite ranges of external control parameters. The present study offers a view complementary to the stationary performance measures analysis and is based on specific realisations of the dynamics. Such an approach is mandatory for the study of the time dependent response to perturbations around the "laminar" production flow regimes and other transient behaviors.

\section{References}

[1] B. Zimmern. Etude de la propagation des arrèts aléatoires dans une chaîne de production. Rev. de Stat. Appl., 4:85-104, 1956.

[2] Y. Dallery and S. B. Gerschwin. Manufacturing flow line systems: A review of models and analytical results. Queueing Systems: Theory and Appl.(QUESTA), 12:3-94, 1992.

[3] J.A. Buzacott and J.G. Shanthikumar. Stochastic models of manufacturing systems. Prentice Hall, Englewood Cliffs, New Jersey, 1993.

[4] W. Henk J.G. Shanthikumar, D.D. Yao and M. Zijm. Stochastic modeling and optimization of manufacturing systems and supply chains. Kluwer Academic Publisher, Bosten, Dordrecht, London, 2003.

[5] D. Chowdhury, L. Santen, and A. Schadschneider. Stochastical physics of vehicular traffic and some related systems. Physics Reports, 329:199-329, 2000.

[6] D. Helbing. Traffic and related self-driven many-particle systems. Reviews of Modern Physics, 73 (4):1067-1141, 2001.

[7] S.P. Sethi and Q. Zhang. Hirarchical Decision Making in Stochastic Manufacturing Sytems. Birkh'auser, 1994.

[8] M. Bando, K. Hasebe, and A. Sugiyama A. Nakayama, A. Shibata. Dynamical model of traffic congestion and numerical simulation. Physical Review E, 51:1035, 1995.

[9] H. Hayakawa and K Naganishi. Theory of traffic jam in one lane model. Physical Review E, 57:3839-3845, 1998.

[10] T. Nagatani. Traffic jams induced by fluctuation of a leading car. Physical Review E, 61;4:3534-3540, 1999.

[11] M.-O. Hongler. Chaotic and Stochastic Behaviour in Automatic Production Lines.p.68. Springer Verlag, 1994.

[12] P. Coillard and J.-M. Proth. Sur l'effet des stocks tampons dans une fabrication en ligne. Rev. Belge Stat. Inf. et R.O., 24:1, 1983. 
[13] K. Konishi, H. Kokame, and K. Hirata. Coupled-map car-following model and its delayed-feedback control. Physical Review E, 60:4000-4007, 1999.

[14] O.L.R. Jacobs. Introduction to Control Theory. Oxford University Press, 1973. 